EUROPEAN ASSOCLATION

FOR THE STUDY OF DIABETES
ASSOCIATION EUROPÉENNE

POUR L'ÉTUDE

DU DIABETTE
EUROPÄISCHE

GESELLSCHAFT FÜR

DIABETOLOGIE

\title{
ORGANIZATION SECTION
}

\section{OFFICERS}

K. LUNDBaEK, Aarhus (retires 1971)

Vice-presidents: E. MARTIN, Geneva (retires 1969)

A. Czyzyk, Warsaw (retires 1969)

Past president: F.G. Youva, Cambridge
President:

Secretary: A.E. Renold, Geneva (retires 1970)

Treasurer: E.F. PFEIFFER, Ulm/D. (retires 1969)

Editor-in-Chief of Diabetologia:

K. OBERDISSE, Düsseldorf (retires 1972)

\section{COUNCIL}

TERM EXPIRING 1969

P.A. Bastente, Bruxelles

T. A. Fraster, London

A. Loubatiteres, Montpellier

I. Pavfer, Bucarest

\author{
TERM EXPTRING 1970 \\ J.L. R-CANDELA, Madrid \\ M. Coppo, Modena \\ W. Creutzhend, Göttingen \\ D. Irkos, Athens \\ J. Poulswa, Copenhagen.
}

TERM EXPIRING 1971

W.J.H. BUTTERFIELD, London

E.R. Frowsch, Zürich

V. Schliack, Berlin

H.D. SöLrNG, Göttingen

\section{HONORARY MEMBERS}

C. H. Best, Toronto - H.C. HaGedorn, Copenhagen

B.A. Houssay, Buenos Aires - R.D. Laweence $\dagger$, London

\section{SUPPORTING MEMBERS}

Ames Europe, Slough, Buckinghamshire - Bayer, Leverkusen - Boehringer, Mannheim · Chemie-Grünenthal, Stolberg · Ciba, Basel - Carlo Erba, Milano - Geigy, Basel - Hoechst, Frankfurt - Hoffmann-La Roche, Basel - Horlicks, Windsor, Berkshire - Hormonchemie, München - Nordisk Insulinlaboratorium, Copenhagen · Novo, Copenhagen · Organon, Oss · Pfizer Europe, Bruxelles - Sandoz, Basel - Schering, Berlin · Vitrum, Stockholm

\section{European Association for the Study of Diabetes Association Européenne pour l'Etude du Diabète Europäische Gesellschaft für Diabetologie}

Draft-Minutes of the 4th Meeting of the General Assembly of the European Association for the Study of Diabetes,

held in Auditorium A, Sciences Building No. 492.11 of the Catholic University of Louvain, Belgium, on Tuesday, 23rd July, 1968 at 11.45

Present: Dr. F.G. Young (President)

Dr. K. LundBaek (Vice-President)

Dr. E. Martin (Vice-President)

Dr. K. OBERDISSE (Editor-in-Chief, Diabetologia)

Dr. E.F. PheIfter (Treasurer)

Dr. A.E. Revold (Secretary)

and approximately 120 members.

\section{Minutes}

The President reported that the Minutes of the Third General Assembly had been printed in Diabetologia 4, No. 3, 1969, page 169 , and inquired if they might be taken as correct. The General Assembly having signified its approval, the President signet the file copy as an accurate record.

$$
\text { 2. Matters Arising }
$$

There were none.

a) President

3. Reports

The President said that in his opening remarks at the start of the Association's meeting he had stated his expectation of the success of the meeting, and he was sure that members would agree that his hopes had been fully justified. Through the hospitality of the University authorities members had been able to see both ancient and modern Louvain, and everyone had cause to be grateful to the Organising Committee for their excellent work.

Tribute had been paid at the opening of the meeting to the late President, Dr. Josfpr Preren Hoet, when 
the Members of the Conference had stood in silence in his memory.

The President expressed the thanks of all members to all those who had contributed to the success of the meeting, especially those supporting members whose names had been printed on the last page of the official pro gramme; he mentioned in particular the contribution of NOVO Industri A/S through whose generosity the translation and printing of the Abstracts had been again made possible in a practical form; their photographic reproduction of slides would also be a most valuable service.

Turning to the affairs of the Association, the President paid tribute to the continued excellent work of the Officers. Despite his absence abroad for much of the last year, Dr. Renowd had been able to exercise good and careful control of the administration, and thanks were also due to Mme LeIsI for her secretarial work in Geneva. Without anticipating the report of the Honorary Auditors, the President felt sure that he could say that the finances of the Association were in good shape, thanks to the work of Dr. Presfrfer. Dr. Oberdisse, as Editor-in-Chief of Diabetologia, had maintained the high standard of publication that members had come to expect.

The Minkowski Award Committee had awarded the Prize for 1968 to Dr. Lars A. CarLson and the wisdom of their choice had been amply confirmed by the excellent lecture delivered on the previous day.

The President reported with pleasure that through the support promised by French Pharmaceutical firms a Claude Bernard Lecture would be delivered at the 5th Meeting of the Association. The Council hoped that this would become an annual feature of the Association's meetings. The form of the Lecture would be somewhat different from that related to the Minkowski Award, and there would be no age limit in the selection of the Lecturer, who would be nominated by the Council.

Finally, the President said that he was happy to report that the Association was generally flourishing.

The President then called on Dr. E.F. PFEIFEER, the Treasurer, to give his report.

\section{b) Treasurer}

Dr. PfETFFer reported that the Opening Balance at 30 June, 1967 was 24538.00 Swiss Franes. 'The year's balance, working up to 30 th June, 1968 was as follows:

Add: Credits Balance 30.6.67 24538.00

Less: Debits $1.7 .67-30.6 .68$

101270.50

Investments

Purchased during year

Balance at bank 30.6 .68

35068.50

66202.00

27776.00

$\overline{38426.00}$ Fr.S.

The Investments Account was reported as follows:

Investments as at 30.6 .67

42822.60

Purchased during year

27776.00

Investments at cost as at $\mathbf{3 0 . 6 . 6 8}$

70598.00 Fr.S.

The Treasurer pointed out that the annual subsriptions received from members were small, and in view of the increasing expenses which faced the Association in the publication of Diabetologia, the continued support of firms was vital.

The Council would be considering the best ways in which the aims of the Association for the furthering of research and intra-European contacts could be achieved with the sums of money at present at their disposal.
The President thanked Dr. Preifrer for his oncouraging report, and called on the Honorary Auditors to make their report.

\section{c) Honorary Auditors}

Dr. J. SCHLTCHTKRULL, on behalf of himself and Dr. J.L. Rodrigukz-Minon, stated from their examination of the statements presented to them the Accounts were in good order, and discharged the Treasurer from responsibility for the year ended 30.6.68.

The President thanked the Honorary Auditors for their services, and called on the Secretary to present his report.

\section{d) Secretary}

The Secretary said that one year ago the membership had been reported as 1046 , which was approximately the number printed in the Membership List. The true figure now, taking into account new members and the regularisation of the situation of members of national associations, particularly the German one, was 1517.

The Membership list contained some errors, and these would be correted by the publication at intervals of amendments and additions. A new revised list would be published about 1970. The Secretary pointed out that unless the green application form was completed, there was no official record in the file of the Association, and urged all members to ensure that the form was completed for future applicants or whenever requested.

An increasing number of subscriptions were now paid through national Associations, but this sometimes resulted in members receiving reminders when their sub. scritption had already been paid. The Secretary craved the indulgence of members if this situation arose.

Dr. RENoLD said that much of the work of the Secretariat was concerned with the publication of the $\mathrm{Ab}$ stracts for the meetings. Authors were particularly requested to check the English versions of these, which would be published in Diabetologia. Some abstracts would not be printed as they were uninformative.

The Secretary reiterated the President's thanks to NOVO Industri A/S for their great help with the translation and publication of the Abstracts.

Turning to the organisation of the present meeting, the Secretary emphasised that more than half of the participants had failed to register by the required date. This made the work of the local Committee very much more difficult. Dr. Renoln took this opportunity to stress the important part played by the local organising Committee, and expressed the Association's thanks to Drs. CoxarD and Hoet, and also to Mme Tulkens who had so efficiently and cheerfully handled the accomodation and transportation arrangements. His remarks were greeted with loud applause.

Continuing, the Secretary reported the existence of a European Diabetes Epidemiology Study Group, which the Council had agreed should be affiliated to the Association, although the exact form which that affiliation should take had yet to be decided. The Chairman of the Group was Dr. V. ScHLIACK, Berlin, DDR, and the Secretary was Dr. R.J. JARkeTT, London, U.K. The Group would report at intervals to the Council, to the annual scientific meetings, and would presumably published reports in Diabetologia. This affiliation created a precedent which might be followed by other Groups concerned with specific problems, and would ensure co-ordination of such investigations within Europe.

The Secretary reported that the 5th Annual Meeting would be held in Montpellier from 15th-18th September, 1969. Future meetings thereafter had been tentatively fixed for:

Warsaw 1970

Cambridge 1971

Madrid 1972 
Although the General Assembly had earlier voted by a show of hands at the 2nd General Assembly in Aarhus that meetings should be held in the early part of July in each year, it had since been found that adherence to this proposal created unforeseen difficulties in some places, and the Secretary asked if the General Assembly had any specific objections to meetings being held later in the year, probably during September. There were no dissent. ing vices from this suggestion.

Finally, Dr. RENoLd paid a warm tribute to his secre. tary, Mme S. LiEISr, who had managed. the affairs of the Secretariat most admirably despite his own absences, and also thanked Mr. J.G.L. JACKSON for all his valuable help with the Minutes of the meetings. Mme LEIsI repeatedly benefited from the advice and help of Dr. DanLex PoMETTA, of Geneva.

The President thanked Dr. RENOLD for his report, and called on Dr.K. OBERDISSE, Editor-in-Chief, Diabetologia, to give his statement.

\section{e) Editor-in-Chief, Diabetologia}

Dr. OBERDisse reported that Volumes 3 and 4 of Diabetologia had consisted of 6 issues, compared with only 4 issues in the previous Volumes. The number of subscribers had increased to 1258 , many of whom came from the United States, probably because of the special arrangement with the American Diabetes Association. All members of the European Association were urged to subscribe to the journal.

The number of manuscripts received had continued to increase, and it was estimated that in 1968 between $80-90$ papers would be published. The size of the journal was to be increased in 1968, and again in 1969 , and this would mean a revision of the subscription rate, which would in future be $100 \mathrm{DM}$ for non-members and $50 \mathrm{DM}$ for members of the European Association. A preferential price $(\$ 15.00)$ would be maintained for members of the American Diabetes Association.

The majority of the manuscripts received had to be returned for corrections, and Dr. OBERDISSE again stressed that manuscripts must be submitted in triplicate, which considerably shortened the time between submis: sion and publication.

As always, the Editors were happy to receive manuscripts from members of the European Association, of the International Diabetes Federation and of national Associations, but Dr. OBERDISSE stressed that the Chairman or President of the national Association was responsible for the style and content of Abstracts submitted from their country, some of which were deplorable.

The names of the referees, without whose expert help publication would be very difficult, would be published annually in the final number for that year.

To the translators and language editors, Drs. David Young, A. Loubatileres, H. Daweke, and H. LIEBERMEISTER, the Editor-in-Chief expressed his sincere thanks for their invaluable help. Dr. OBfrRdisse paid tribute to the outstanding help given by Dr. Loubatrikres, the retiring Associate Editor, who would be succeeded by Dr. V. Conard.

The General Assembly noted the appointment of the following persons as members of the Advisory Board in succession to Drs. Randle, Azerad, R-Candeta, SkraBATO and WALAAS, who retired by rotation:

\author{
Dr. A. CzyzyK \\ Dr. H. G. LIPPMANN \\ Dr. P. Mitathe \\ Dr. E.A. NrkKILÄ \\ Dr. C.N. Hates.
}

Finally, the Editor-in-Chief thanked the Officers of the Association and his colleagues of the Editorial and Advisory Boards for their unfailing support, and the publishers for their constant efforts to produce an attractive journal.

The President thanked Dr. OBendisse for his report.

The President then invited any discussion from the audience on the five Reports given. There being no questions, the President declared the Reports adopted.

\section{a) Council}

\section{Elections}

There being no other nominations from the Assembly, the President declared the following persons nominated by the Council elected as members of the Council for the period 1968-1971:
Dr. E.R. Froesch, Zurich
Dr. H.D. Söling, Göttingen
Dr. V. Schliack, Berlin, DDR.

The President also declared the following persons elected as members of the Council to take office immediately following the 5th General Assembly, for the period $1969-1972$ :
Dr. V. Conard, Brussels
Dr. M. Dérot, Paris
Dr. I. MAGYAR, Budapest
Dr. P.J. RandLe, Bristol
Dr. K. SchöFruing, Frankfurt/M.

\section{b) Honorary Auditors}

Drs. J. SChLICHTKRULL and J.L. RodRIGUEz-Minon were elected as the Honorary Auditors for the following year.

\section{Any other business}

Dr. Loubatrìnes gave advance welcome to the Association to Montpellier for their 5th Annual Meeting from 15th-18th September, 1969. Montpellier is the oldest School of Medicine still in existence and dates from the twelfth century. His remarks were greeted with loud applause.

\section{Handing over of the Presidency}

Dr. Young first reported that because of the election of Dr. LuNDBAEK as the 3rd President of the Association, a vacancy existed in one of the two offices of Vice-President. The Council had appointed Dr. A. Czyzyk to this position for the period 1968-1969.

Dr. Youna said that he was deeply grateful for the honour paid to him in inviting him to become the 2nd President of the Association. He had greatly enjoyed his term of office, and he could not have been more fortunate in his colleagues, from whom he had received great help and kindness. Especially he wished to thank Dr. RENOLD for his effective and discrete assistance at all times. Telephone calls between Geneva and Cambridge would now diminish, but the ,hot line" would doubtless be transferred to between Geneva and Aarhus!

Dr. Young said that he was particularly happy to hand over the Presideney to a distinguished clinician, and he was sure that ander Dr. LuNDBAEK's leadership, the Association would continue to go from strength to strength.

To great applause, Dr. Young then welcomed Dr. LUNDBAEK to the Presidency.

Thanking the General Assembly for the great honour, Dr. LUNOBAEK said that he would try to do his best. The Association had been very fortunate in its 2nd President, who had set a fine example to follow, and on behalf 
of the Association Dr. LuNDBAEK sincerely thanked Dr. Young for his years of service and example. The President's remarks were greeted with prolonged applause.

Finally, Dr. LondBaEk said that he had no new presidential policy to announce. He hoped that the Association would continue along the same lines as already firmly established, namely the publication of Diabetologia and the arranging of annual meetings which were interesting, factual and unceremonious. In all this he would continue to rely on the invaluable help of the Officers of the Association, and in particular the indefatigable Secretary, Dr. RENOLD.

Dr. K. LundBaEK

President
Dr. A E. RmiNOLD Secretary

\section{Projet de Procès-Verbal de la 4ème Assemblée Générale de l'Association Européenne pour l'Etude du Diabète} du 23 juillet 1968, à 11 h 45 à l'Université Catholique de Louvain Belgique. Auditoire A. Bâtiment No. 492.11

\author{
Présents: Dr. F.G. Young (Président) \\ Dr. K. LundBaEK (Vice-Président) \\ Dr. E. MarTin (Vice-Président) \\ Dr. K. Oberdisse (Rédacteur-en-Chef Diabeto- \\ logia) \\ Dr. A.E. RENoLd (Socrétaire) \\ et environ 120 membres.
}

\section{Procès-Verbal}

Le Président informe que le procès verbal de la 3ème Assemblée générale a été publié dans Diabetologia 4, No. 3, page 169,1968 , et en demande l'approbation par l'Assemblée générale. Formellement approuvé par cotte dernière, le procès verbal est signé par le Président.

\section{Propositions résultant du procès-verbal} aucune

\section{Rapports}

\section{a) Rapport du Président}

Le Président rappelle ses remarques lors de l'ouverture. II avait prédit le succès de cette réunion de Louvain et cet espoir s'est avéré entièrement justifié. L'hospitalité des Autorités universitaires a permis aux membres de l'Association de connaittre dans les meilleures conditions le Louvain ancien et le Louvain moderne. L'Association est en outre très reconnaissante au Comité d'organisation local de l'excellence de toutes les dispositions prises.

Le Président rappelle encore que tous les membres présents à la séance d'ouverture ont rendu hommage à leur Président défunt, le Professeur JosePH PIERRE HoEt, en observant une minute de silence en sa mémoire.

Au nom de tous, le Président remercie les membres bienfaiteurs qui ont contribué et collaboré au succès de la réunion; leur liste est publiée à la dernière page du programme officiel. II remercie plus spécialement la maison NOVO Industri A/S de sa contribution aux frais de traduction et d'impression des résumés, ainsi que de l'innovation que représente son excellent service de reproduction des diapositives.

En commentant les affaires de l'Association, le Président remercie avant tout les membres du Comité de direction et des diverses commissions de leur appui. Le Dr. Renoud, malgré son année sabbatique à l'étranger, a réussi à suivre de loin les affaires administratives de l'Association, grâce à l'appui de Mme LEIsI du Secrétariat de Genève, qu'il remercie également. Sans anticiper sur le rapport des vérificateurs des comptes, lo Président pense pouvoir confirmer le bon ordre et l'état satisfaisant des finances de l'Association, et se plait à reconnaître I'efficacité du Dr. PFEIFFER. Le Dr. OBERdisse, rédacteur-en-chef de Diabetologia, a réussi à maintenir le haut niveau des publications dans cette revue.

Le Comité du Prix Minkowski a décerné le prix de 1968 au Dr. LaRs A. CarLson de Stockholm, et la très belle leçon déjà présentée par le lauréat a entièrement justifié le choix du Comité.

Le Président informe que l'appui de maisons pharmaceutiques françaises permettra la mise au programme, lors de la 5ème réunion de l'Association, d'une Leçon Claude Bernard dont le Comité espère qu'elle deviendra annuelle. Cette Leçon, de synthèse surtout, sera quelque peu différente de celle du Prix Minkowski. Lee choix du lauréat incombera au Comité de direction. Il n'y aura pas de limite d'âge.

Le Président conclut par la constatation que les affaires de 1'Association lui donnent un excellent certificat de santé!

Le Président invite le Dr. PFenfrer à présenter son rapport.

\section{b) Rapport du Trésorier}

Le Dr. PFEIFrer rappelle qu'en date du 30 juin 1967 , le solde au bilan de l'Association était de Fr.S. 24538.00 . Le bilan au 30 juin 1968 se présente comme suit:

\begin{tabular}{|c|c|c|c|}
\hline Plus: & $\begin{array}{l}\text { Solde au } 30 \text { juin 1967: } \\
\text { Crédits du 1.7.67 au 30.6.68 }\end{array}$ & $\begin{array}{l}24538.00 \\
76732.00\end{array}$ & \\
\hline \multirow[t]{3}{*}{ Moins: } & Débits du 1.7.67 au 30.6 .68 & & $\begin{array}{r}101270.50 \\
35068.50\end{array}$ \\
\hline & Investissements en cours d'ar & nnée & $\begin{array}{l}66202.00 \\
27776.00\end{array}$ \\
\hline & Solde en banque au $\mathbf{3 0 . 6 . 6 8}$ & Fr.s. & 38426.00 \\
\hline
\end{tabular}

Le compte des investissements est établi comme suit:

Investissements au 30.6 .67 42822.60

Investissements en cours d'année 27776.00

Investissements au 30.6 .68 $\overline{70598.00}$ Fr.S.

Le Trésorier rappelle que le montant provenant des cotisations annuelles est relativement faible et qu'en tenant compte des dépenses de l'Association pour Diabetologia, la continuation de l'appui des maisons pharmaceutiques reste essentielle.

Le Comité de direction devra on outre s'attacher plus particulièrement à définir la façon la meilleure pour I'Association de promouvoir la recherche et les échanges intra-européens avec les sommes actuellement à sa disposition.

Le Président remercie le Dr. PfenFFER de son rapport et prie les vérificateurs des comptes de présenter le leur.

\section{c) Vérificateurs des comptes}

Les Drs. J. SchtTchtKRULl et J.L. RodRIGUezMINON confirment que l'examen du bilan soumis montre que les comptes de l'Association européenne pour l'étude du diabète sont en bon ordre et que l'Assemblée générale 
peut donner décharge au Trésorier pour l'année se terminant.

Le Président remercie les vérificateurs des comptes et invite le Secrétaire à présenter son rapport.

\section{d)Rapport du Secrétaire}

Le Secrétaire informe que, l'année dernière, l'Association comptait 104.6 membres, ce qui correspond à peu près au nombre figurant dans la liste des membres qui a été publiée ce printemps. En tenant compte des nouvelles admissions et de la régularisation du statut des membres d'associations nationales, de l'Association allemande surtout, le nombre effectif est porté à 1517 .

La liste des membres comporte quelques erreurs qui seront corrigées au cours de la publication régulière des additions et des mutations. Une nouvelle liste complète, revue et corrigée sera probablement publiée vers 1970 . Le Secrétaire rappelle que l'Association ne possède pas de documentation valable chaque fois que le questionnaire sur papier vert n'a pas été rempli. II prie instam. ment tous les membres de veiller à ce que ce questionnaire soit retourné au Secrétariat dûment rempli, chaque fois que la demande en est faite.

Une proportion croissante des cotisations est réglée par l'intermédiaire des Associations nationales ou régionales, ce qui est réjouissant. Pourtant, il en résulte parfois qu'un membre reçoit un rappel pour une cotisation déjà versée. Le Secrétaire prie les membres d'excuser ces erreurs difficiles à éviter.

Le Dr. Renold rappelle l'activité importante du Secrétariat pour la publication des résumés présentés pour les réunions. Il prie les auteurs de revoir la version anglaise des résumés, destinée à être publiée dans Diabetologia. Certains résumés peu informatifs ne seront pas publiés. Il réitère les remerciements du Président à la maison NOVO Industri A/S pour l'appui donné à la traduction et à la publication des résumés.

En considérant l'organisation de la réunion de Louvain, le Secrétaire constate que bien plus de la moitié des participants ne se sont inscrits que longtemps après la date fixée, contribuant par là à la très grande et inutile complication des travaux du Comité d'organisation. Le Dr. RENOLD saisit l'occasion pour remercier les organisateurs, les Drs. V. Conard et J.J. Hoet, ainsi que Mme TutKkens de son appui dynamique et généreux à l'organisation complexe des transports et des logements. Ces dernières remarques provoquent les vifs applaudissements de l'Assemblée.

Le Secrétaire informe en outre qu'il existe un groupe de travail européen consacré à l'étude de l'épidémiologie du diabète. Ce groupe de travail désire étre reconnu par l'Association et lui être affilié. Le Comité de direction ne voit aucune raison de s'opposer à une telle affiliation, dont le mode exact reste à définir. Ce groupe de travail est présidé par le Dr. V. Schliack; son secrétaire est le Dr. R.J. JARReTT. II rapportera de temps en temps au Comité sur ses activités, sans doute aussi lors des réunions annuelles, ainsi que dans Diabetologia. Cette affiliation crée un précédent heureux et il est probable que d'autres groupes créés pour l'étude de problèmes spécifiques désireront une affiliation semblable, permettant une meilleure coordination de leurs activités sur le plan européen.

Le Secrétaire confirme que la 5ème réunion annuelle se tiendra à Montpellier, France, du 15 au 18 semptembre 1969. Pour les années suivantes, les réunions sont provisoirement prévues à

$$
\begin{aligned}
& \text { Varsovie en } 1970, \\
& \text { Cambridge en 1971, } \\
& \text { Madrid en 1972. }
\end{aligned}
$$

Bien qu'il ait été décidé lors de la 2ème assemblée générale à Aarhus de retenir, en règle générale, des dates pendant la première quinzaine de juillet pour les réunions annuelles, il s'est avéré que des difficultés imprévues se présentent souvent et qu'il n'est pas toujours possible d'adhérer à cette décision. Le Secrétaire demande à l'Assemblée générale s'il y a objection grave à prévoir fréquemment des réunions plus tard dans l'année, en septembre par exemple. Aucune objection n'est faite à ce projet.

Enfin, le Dr. RunoLd remercie très chaleureusement sa secrétaire du Bureaur de Genève, Mme LEISI, et rappelle avec quelle conscience elle s'est occupée des affaires de l'Association pendant son absence. Il adresse ses remerciements à M. J.G.L. JACKson de continuer de se charger de la rédaction des procès verbaux des réunions du Comité et de l'Assemblée. Pendant l'absence du Secrétaire, Mme LEIsI a souvent eu l'occasion de bénéficier des conseils et de l'appui du Dr. Danizu Pometta de Genève.

Le Président remercie le Dr. Revold de son rapport et prie le Dr. OвFRDisse de donner un aperçu de ses activités en tant que Rédacteur-en-chef de Diabetologia.

\section{e) Rapport du Rédacteur-en-chef de Diabetologia}

Le Dr. K. Oberdisse informe que les volumes 3 et 4 de Diabetologia comportent 6 numéros chacun alors que les deux premiers $n^{\prime}$ en comportaient que 4. Le nombre des abonnés a augmenté à 1258 , augmentation qui a surtout porté sur les abonnés aux Etats-Unis, ceci probablement grâce aux conditions spéciales offertes aux menabres de l'American Diabetes Association. Le Rédacteur-en-chef invite encore une fois tous les membres de notre Association à s'abonner à Diabetologia.

Le nombre de manuscrits reçus a augmenté et il est prévu que de 80 à 90 articles seront publiés en 1968. En 1968 et surtout en 1969 , le nombre de pages sera sensiblement augmenté, ce qui entraînera un nouveau tarif d'abonnement. Celui.ci sera, dès 1969 , de DM 100. - pour les non-membres, et de DM 50.- pour les membres. Un prix spécial pour les membres de l'American Diabetes Association sera maintenu: il sera de $\$ 15.00$.

Un très grand nombre de manuscrits a dû être retourné pour corrections et le Dr. ObERdisse prie de nouveau tous les auteurs de les lui faire parvenir en trois exemplaires, ce qui permet de diminuer considérablement le délai entre la soumission et la publication des articles.

Les rédacteurs continuent à être heureux de recevoir des manuscrits provenant des membres de notre Association, de ceux de la Fédération Internationale du Diabète et des Associations nationales, mais le Dr. OrkiDISsE rappelle que les Présidents des Associations nationales assument la responsabilité stylistique et qualificative des résumés de présentations faites dans le cadre de réunions nationales, résumés souvent fort mal présentés.

Dorénavant, la liste des experts ayant contribué à l'appréciation des manuscrits soumis sera publiée en. dernière page du numéro final de chaque volume.

Le Dr. OBERDISSE adresse ses remerciements sincères aux traducteurs et rédacteurs linguistiques, soit aux Drs. D. A.B. Young, A. Loubatikres, H. Dawerke et H. LIEBERMEISTER. Il remercie chaleureusement le Dr. A. LouBATIÈRES, rédacteur sortant, de son appui constant et dévoué. Le Dr. LoubatièRes sera remplacé par le Dr. V. CONARD.

Les nominations suivantes au Comité de lecture, en remplacement des Drs. P.J. RANDLE, E. AziERAD, J.L. R-Candera, Z. Skrabato et $O$. Watias, sont soumises à l'attention de l'Assemblée générale:

$$
\begin{aligned}
& \text { Dr. A. CZYZYK } \\
& \text { Dr. H.G. LIPPMaNN } \\
& \text { Dr. P. MralHE } \\
& \text { Dr. E.A. NHKKḦ̈ } \\
& \text { Dr. C.N. HAJES. }
\end{aligned}
$$


Le Rédacteur-en-chef remercie ses collègues des Comités de rédaction et de lecture de leur assistance continue et bienveillante, ainsi que la maison Springer des efforts voués à la production de la revue. port.

Le Président remercie le Dr. Oberdisse de son rap-

Le Président invite l'Assemblée généralo à discuter l'ensemble des cinq rapports présentés. En l'absence de discussion, le Président, au nom de l'Assemblée, déclare que ces rapports sont approuvés.

\section{Elections}

a) Comité de Direction

N'ayant reçu aucune autre proposition de la part de l'Assemblée générale, le Président, au nom de cette dernière, confirme l'élection des candidats présentés par le Comité sortant:

Pour la période de 1968 à 1971 :

Dr. E.R. FrowsCh, Zurich

Dr. H.D. SörxNG, Göttingen

Dr. V. Schlraok, Berlin, DDR.

Les membres suivants sont élus au Comité de direction pour la période de 1969 à 1972, avec entrée en fonction après la 5ème Assemblée générale:

Dr. V. Conard, Bruxelles

Dr. M. DÉrot, Paris

Dr. I. MagYaR, Budapest

Dr. P.J. Randee, Bristol

Dr. K. SoHöFFIING, Frankfurt/M.

b) Vérificateurs des comptes

Les Drs. J. SchlichtkruLL et J.L. RodriguezMrnos sont élus vérificateurs honoraires des comptes pour l'année 1968-1969.

\section{Divers}

D'avance, le Dr. Loubatitizes souhaite la bienvenue à l'Association pour sa prochaine réunion annuelle à Montpellier, France, du 15 au 18 septembre 1969, soit donc à la plus ancienne Faculté de médecine encore en activité continue! Ces remarques sont accueillies par les applaudissements nourris de l'Assemblée générale.

\section{Transfert de la Presidence}

Au préalable, le Dr. F.G. Yourg signale la charge de Vice-Président qui est à repourvoir de par la nomination du Dr. K. Lundbaek à la Présidence de l'Association. Le Comité de direction a désigné un deuxième VicoPrésident en la personne du Dr. A. Czyzyk pour la période de 1968 à 1969.

Le Dr. F.G. Young exprime alors sa gratitude à l'Association de lui avoir conféré l'honneur d'être son 2ème Président. Il s'estime heureux d'avoir pu servir l'Association pendant trois années et $n$ 'aurait pu souhaiter avoir des collègues plus prévenants et plus coopératifs que ceux dont il se sépare aujourd'hui. Il retrace avee beaucoup d'humour l'appui discret et efficace que lui a donné le Dr. Renold à tout moment. Il évoque les entretiens téléphoniques entre Genève et Cambridge, qui iront en diminuant, mais il ne doute pas que la «ligne chaude» sera maintenue entre Genève et Aarhus!

Le Dr. F.G. Young exprime sa satisfaction de pouvoir remettre la Présidence de notre Association entre les mains d'un éminent clinicien, le Dr. K. LUNDBAEK. Il est convaincu que, sous la présidence de ce dernier, l'Association se développera avec force et vigueur. Sous les applaudissements de l'audience, le Dr. Young transmet la Présidence au. Dr. Lundbaek.

Le Dr. LuNDBAEK remercie l'Assemblée générale du grand honneur qu'elle lui confère et affirme que c'est de son mieux qu'il essayera de s'acquitter de sa tâche.

L'Association a été très fortunée d'avoir eu en son deuxième Président, le Dr. F.G. Young, une personnalité dont la fermeté, la probité et le dynamisme serviront toujours de modèle. Au nom de l'Association, le Dr. LUNDBAER remercie très sincèrement le Dr. F.G. YouNG de tout ce qu'il a fait pour notre Association, remerciements qui sont soulignés par les applaudissements des membres prósents.

Le Dr. LUNDBAerr n'a pas de nouvelle politique présidentielle à annoncer. Il souhaite que l'Association continue la ligne d'activité déjà tracée, en publiant Diabetologia et en réalisant des réunions annuelles intéressantes, efficaces et incérémonieuses. A cet effet, il continuera à s'appuyer sur l'aide des autres membres du Comité de direction, surtout sur celle de l'infatigable Secrétaire, le Dr. RENOLD.

Dr. K. LUNDBAEK

Président
Dr. A.E. Renotd Secrétaire

\section{Protokoll-Entwurf über die 4. Generalversammlung der Europäischen Gesellschaft für Diabetologie im Auditorium A, Studiengebäude Nr.492.11 der Katholischen Universität Louvain, Belgien, 23. Juli 1968, 11.45 Uhr}

Anwesende: Dr. F.G. YouNG (Präsident)

Dr. K. LunDBaEK (Vizepräsident)

Dr. E. Martin (Vizepräsident)

Dr. K. Oburdisse (Herausgeber, Diabetologia)

Dr. E.F. PfetheEr (Schatzmeister)

Dr. A.E. RENoLd (Sekretär)

und ungefähr 120 Mitglieder

\section{Protokoll}

Der Präsident berichtet, daß die Protokolle der 3. Generalversammlung in Diabetologia 4, Nr. 3, 1968, Seite 169, veröffentlicht wurden und erkundigte sich, ob ihre Richtigkeit anerkannt werden könne. Nach Zustimmung der Versammlung, zeichnet der Präsident das zur Ablage bestimmte Exemplar als ordnungsgemäßen Bericht.

\section{Besondere Angelegenheiten}

Keine

\section{Berichte}

a) Präsident

Der Präsident wies darauf hin, daß seine in der Er. öffnungsrede zu Beginn der Versammlung geäußerten Hoffnungen über den Erfolg des Treffens sicherlich von den Mitgliedern in vollem Umfang geteilt würden. Die Gastfreundschaft der Vertreter der Universität ormög. lichte es, das ,alte" und das ,neue ${ }^{\prime c}$ Louvain kennenzulernen, und jeder hatte Veranlassung, dem Organisationskomitee für seine ausgezeichnete Arbeit zu danken.

Zu Beginn des Treffens erhoben sich die Teilnehmer des Kongresses schweigend im Gedenken um die Verdienste des verstorbenen Präsidenten, Dr. JosEPH PlerRE 
Hozx. Im Namen aller Teilnehmer dankte der Präsident all denen, die zum Erfolg des Treffens beigetragen hatten, besonders den unterstützenden Mitgliederm, deren Namen auf der letzten Seite des Programms aufgeführt waren. $\mathrm{Ex}$ wies besonders auf den Beitrag der Firma NOVOIndustrie A/S hin, durch deren GroBzügigkeit die Übersetzung und der Abdruck der „Abstracts" erneut ermöglicht wurde. Ihre Fotokopien der Diapositive würden ebenso eine äußerst wertvolle Hilfe sein.

Zurückkommend auf die Angelegenheiten der Gesellschaft, dankte der Präsident anerkennend der ausgezeichneten Arbeit des Beirates und des geschäftsführenden Vorstandes. Trotz seiner Abwesenheit im Ausland während fast des ganzen letzton Jahres ermöglichte es Dr. RæiroLD, die Verwaltungsangelegenheiten sorgfältig zu führen. Ebenso dankte er Frau Lwisr für ihre Sekretariatsarbeit in Genf. Ohne den Bericht der Kassenprüfer vorwegzunehmen, versicherte der Präsident, daß es um die finanziellen Angelegenheiten der Gesellschaft gut bestellt sei dank der Arbeit von Dr. PHEIATER. Dr. OberDISSE, als Herausgeber der Diabetologia, hatte das hohe Niveau der Veröffentlichungen bestens aufrechterhalten können.

Das Minkowski-Preiskomitee hatte den Preis für 1968 Dr. Lars A. CarLsor zugesprochen und wurde in seiner Wahl durch den ausgezeichneten Vortrag bestätigt.

Der Präsident berichtete mit Freude, daß durch die versprochene Unterstützung durch französische pharmazeutische Firmen ein Claude-Bernard-Ehrenvortrag zum 5. Treffen der Vereinigung gehalten werden würde. Der Vorstand gab seiner Hoffnung Ausdruck, daß dies zu einem alljährlichen Ereignis bei den Treffen der Vereinigung werden könne. Die Form des Festvortrages sollte etwas anders gestaltet sein als bei dem Minkowski-Preis, und es sollte keine Altersgrenze bei der Auswahl der Preisträger geben, die vom Beirat benannt würden.

Schließlich brachte der Präsident seine Zufriedenheit über die allgemeine blühende Entwicklung zum Ausdruck. Anschließend bat er Dr. E. F. PfEIFrER, den Schatzmeister, um seinen Bericht.

\section{b) Schatzmeister}

Dr. PfEnffre berichtete, daß sich die Eröffnungsbilanz am 30.6.67 auf 24538.00 Fr. S. belief. Die Jahresabschlußbilanz bis zum 30.6.68 errechnete sich wie folgt:

Fr.S.

Eröffnungsbilanz 30.6.67 24538.00

Haben: Kredite $1.7 .67-30.6 .68 \quad 76732.50 \quad 101270.50$

Soll: Lasten

66202.00

27776.00

Anschaffnungen während des Jahres

Kassenbilanz 30.6.68

38426.00

Der Geldanlagebericht setzt sich folgendermaßen zusammen:

Anlagen am 30.6.67

Anschaffungen während des Jahres

Anlagen in Bar am 30.6.68

42822.60

27776.00

$\overline{70598.60}$ Fr.S.

Der Schatzmeister hob hervor, daß die jährlichen Beiträge der Mitglieder gering seien, und angesichts der wach. senden Ausgaben, zu denen die Vereinigung durch die Veröffentlichung der Diabetologia veranlaßt wurde, sei die ständige Unterstützung durch Firmen notwendig.

Der Vorstand sollte sich weiterhin überlegen, wie die Ziele der Gesellschaft zur Förderung der Forschung und. intraeuropäischer Kontakte am besten mit den verfügbaren Mitteln erreicht werden könnten.
Der Präsident dankte Dr. PrEIFFER für seinen ermutigenden Bericht und bat die Rechnungsprüfer um ihre Beiträge.

\section{c) Rechnungsprüfer}

Dr. J. SortcichtKRoLI stellte in seinem und im Namen von Dr. J.L. RodRiguez-Minor fest, daß nach Prüfung der ihnen gemachten Angaben die Beträge in Ordnung seien, und entlastete den Schatzmeister von seiner Verantwortung bis 30.6.68. Der Präsident danlkte den Rechnungsprüfern für ihre Dienste und forderte den Sekretär zur Berichterstattung auf.

\section{d) Sekretär}

Der Sekretär teilte mit, daß sich die Mitgliederzahl vor 1 Jahr auf 1046 belief, was annähernd der in den Mitgliederlisten aufgeführten Zahl entsprach. Der jetzige Stand unter Berücksichtigung der neuen Mitglieder und des Ausgleichs durch die Übernahme der Mitglieder aus den nationalen Vereinigungen, besonders der deutschen, ergab 1517.

Die Mitgliederliste enthielt einige Fehler, die durch eine Veröffentlichung über Änderungen und Zusätze in zeitlichen Abständen berichtigt werden sollte. Fine neue und überholte Liste sollte etwa 1970 herausgegeben werden. Der Sekretär hob hervor, daß, solange nicht die offizielle Beitrittsgesuchsform ergänzt würde, eine offizielle Führung in den Akten der Gesellschaft nicht möglich sei, und ersuchte alle Mitglieder nachdrücklich, sicherzustellen, daß die formellen Regeln in Zukunft für alle Bewerbungen beachtet würden.

Eine steigende Zahl von Beiträgen wurde neuerdings durch nationale Verbände erstattet, was einige Male zu Mahnungen führte, obwohl die Beiträge schon erstattet waren. Der Sekretär bat um Nachsicht der Mitglieder, die davon betroffen waren. Dr. Rwrold berichtete, daß ein großer Teil der Arbeit des Sekretariats darin bestand, die Veröffentlichung der, ,Abstracts " der Treffen auszuarbeiten. Die Verfasser wurden besonders ersucht, ihre Artikel ins Englische zu übersetzen, wenn sie in Diabetologia veröffentlicht werden sollten. Einige "Abstracts" wurden nicht gedruckt, da sie ohne Informationswert geweson seien.

Der Sekretär wiederholte den Dank des Präsidenten für die große Hilfe bei Übersetzung und Veröffentlichung der ,Abstracts" durch die Firma NOVO-Industrie A/S.

Bezüglich der Organisation des derzeitigen Treffens betonte der Sekretär mit Nachdruck, daß über die Hälfte der Teilnehmer versäumt hatte, sich zu dem gewünschten Termin anzumelden. Das erschwerte die Arbeit des örtlichen Komitees ganz erheblich. Dr. RENoud wies bei dieser Gelegenheit darauf hin, welch bedeutende Rolle das örtliche Organisationskomitee bei der Beschaffung der Unterkünfte und Organisation der Veranstaltungen spielte, und übermittelte den Dank der Gesellschaft an die Herren Drs. Conard und Howt sowie Fran Tutkens, die besonders hilfreich und freudig mitgeholfen hatte. Die Versammlung stimmte dem Sekretär mit Beifall zu.

Der Sekretär führte weiterhin aus, daß eine Europäische Arbeitsgruppe für Epidemiologie des Diabetes bestünde, die nach Zustimmung durch den Beirat der Gesellschaft regelmäßige Kontakte mit der Gesellschaft pflegen würde, obgleich die Art dieser Kontakte noch zu entscheiden sei. Der Vorsitzende dieser Gruppe ist Dr. V. Sc\#LIACK, Berlin, DDR, der Sekretär Dr. R.J. JARRErT, London, U.K. Die Gruppe sollte in Abständen dem Vorstand während der jährlichen wissenschaftlichen Treffen berichten und vermutlich Arbeiten in Diabetologia veröffentlichen. Diese Angliederung solle als Beispiel für andere Gruppen dienen, die besondere Probleme bearbeiteten. Dies würde die europäische Zusammenarbeit bei besonderen Forschungsvorhaben erleichtern. 
Der Sekretär berichtete, daß die 5. Jahrestagung vom 15.-18.9.1969 in Montpellier abgehalten werden solle. Weitere Treffen wurden vorläufig festgelegt:

\section{0 in Warschau \\ 1971 in Cambridge \\ 1972 in Madrid.}

Obwohl die Hauptversammlung früher einmal (2. Hauptversammlung in Aarhus) durch Handerheben beschlossen hatte, die Versammlungen Anfang Juli jeden Jahres abzuhalten, wurde seitdem gefunden, daß dieses Vorhaben in einigen Fällen unvorhergesehene Schwierigkeiten hervorrief. Deswegen fragte der Sekretär, ob die Hauptversammlung einen Einwand gegen einen späteren Termin im Jahr, im allgemeinen September, habe. Keine abweichende Meinung wurde vorgebracht.

SchlieBlich sprach Dr. RENOLD seinen herzlichen Dank seiner Sekretärin, Frau S. LEISI, aus, die alle Angelegenheiten des Sekretariats in bewunderungswürdiger Weise auch während seiner Abwesenheit erledigt hatte. Ebenso dankte er Herrn J. G.L. JAckson für seine wertvolle Hilfe bei der Abfassung der Protokolle der Tagungen. Frau Leist sei Dr. Daniel Pometra, Genf, für Hinweise und Hilfe zu Dank verpflichtet.

Der Präsident dankte Dr. Renold und bat Dr. $K$. OBERDISSE, den Herausgeber von Diabetologia, um seinen Bericht.

\section{e) Herausgeber, Diabetologia}

Dr. OBERDISSE berichtete, daß Band 3 und 4 der Diabetologia aus je 6 Heften gegenüber 4 in den vorausgegangenen Bänden bestanden hätten. Die Zahl der Abonnenten sei auf 1258 angestiegen, davon viele aus den Vereinigten Staaten, wahrscheinlich durch die besonderen Vereinbarungen mit der Amerikanischen Diabetes-Gesellschaft. Alle Mitglieder der Europäischen Gesellschaft wurden erneut aufgefordert, die Zeitschrift zu abonnieren.

Die Zahl der eingegangenen Manuskripte stieg fort. gesetzt, und es wurde geschätzt, daß 1968 etwa $80-90$ Veröffentlichungen erscheinen würden. Der Umfang der Zeitschrift mußte 1968 erweitert werden und wird 1969 erneut erweitert werden müssen, was eino Änderung der Bezugsgebühren ergeben müßte, nämlich DM 100.- für Nichtmitglieder und DM 50. - für Mitglieder der Europäischen Gesellschaft. Ein Vorzugspreis (\$15.00) sollte den Mitgliedern der Amerikanischen Diabetesgesellschaft weiterhin eingeräumt werden.

Die Mehrzahl der eingegangenen Manuskripte mußte zu Korrekturen zurückgeschickt werden. Dr. ÖBERDISSE wies nachdrücklich darauf hin, daß Manuskripte in dreifacher Ausfertigung eingeschickt werden müßten, was die Zeit zwischen Einsendung und Veröffentlichung beträchtlich verkürze. Wie bisher wären die Herausgeber auch in Zukunft glücklich, Manuskripte von Mitgliedern der Europäischen Gesellschaft, des Internationalen Diabetesverbandes und der nationalen Gesellschaften zu erhalten. Jedoch betonte Dr. OBERDISSE, daß die Vorsitzenden oder Präsidenten der nationalen Gesellschaften für Stil und Inhalt dor ,Abstracts" aus dem eigenen Land verantwortlich seien. Einige dieser ,Abstracts" wären in traurigem Zustand gewesen.

Die Namen der Referenten, ohne deren Fachhilfe eine Veröffentlichung sehr schwierig gewesen wäre, sollten jährlich im letzten Heft veröffentlicht werden.

Den Übersetzern und Sprachbearbeitern, Drs. DAvID Young, A. Loubatikireas, H. Daweke und H. LIEBerRMEISTER, sprach der Herausgeber seinen besonderen Dank für ihre außerordentliche Hilfe aus.

Dr. OBERDISSE dankte der außerordentlichen Hilfe durch Dr. Loubatrìres, dem ausscheidenden Mitherausgeber, der abgelöst werden soll durch Dr. V. ConaRD.
Die Hauptversammlung erfuhr die Ernennung folgender Personen als Mitglieder des Beraterausschusses in Nachfolge von Dr. Randue, Dr. Azmrad, Dr. R-CarDela, Dr. Skrabalo und Dr. Waraas, die im Wechsel ausschieden:

$$
\begin{aligned}
& \text { Dr. A. CZYzYK } \\
& \text { Dr. H. G. LrPPMANN } \\
& \text { Dr. P. MTathe } \\
& \text { Dr. E. A. NtKKIL } \\
& \text { Dr. C.N. HaLES. }
\end{aligned}
$$

Schließlich dankte der Herausgeber seinen Kollegen des Herausgeber- und Beratungsausschusses für ihre unfehlbaren Hinweise und dem Verleger für seine ständigen Bemühungen, eine ansprechende Zeitschrift herauszugeben. richt.

Der Präsident dankte Dr. ObeRdisse für dessen Be-

Der Präsident forderte dann zur Diskussion der 5 Berichte durch die Versammlung auf. Es gab keine Fragen. Der Präsident erklärte die Berichte für angenommen.

\section{a) Vorstand}

\section{Wahlen}

Nachdem keine anderen Nominierungen durch die Versammlung vorlagen, erklärte der Präsident folgende vom Vorstand nominierten Personen als Mitglieder des Vorstandes für die Zeit von 1968 bis 1971 für gewählt:

$$
\begin{aligned}
& \text { Dr. E.R. Frowsch, Zürich } \\
& \text { Dr. H.D. SölTNG, Göttingen } \\
& \text { Dr. V. SoHurack, Berlin, DDR. }
\end{aligned}
$$

Der Präsident erklärte ebenso folgende Personen als Mitglieder des Vorstandes für gewählt, unmittelbar nach der 5. Hauptversammlung in der Zeit von 1969 bis 1972 die Aufgaben zu übernehmen:
Dr. V. Conard, Brüssel
Dr. M. Derot, Paris
Dr. I. MAGYAR, Budapest
Dr. P.J. RaNDLE, Bristol
Dr. K. SoHöFFLING, Frankfurt/M.

\section{b) Rechnungsprüfer}

Drs. J. SChlichtKRunt und J.L. RoDriguez-Minon wurden als Rechnungsprüfer für das folgende Jahr gewählt.

\section{Verschiedenes}

Dr. Lounatières hieß die Gesellschaft im voraus in Monpellier zur 5. jährlichen Tagung vom 15.-18. September 1969 willkommen. Montpellier ist die älteste Medizinschule, die noch besteht, und datiert bis auf das 12. Jahrhundert zurück. Seine Hinweise wurden mit lautem Applaus begrüßt.

\section{6. Übergabe der Präsidentschaft}

Dr. Young berichtete zunächst, daß durch die Wahl von Dr. LundBaek als 3. Präsidenten der Gesellschaft einer der beiden V'izepräsidentenstühle frei würde. Der Vorstand forderte Dr. A. CzyzyK auf, diesen Posten für die Zeit von 1968 bis 1969 zu übernehmen.

Dr. Young führte aus, daß er zutiefst dankbar für die ihm übertragene Fihre sei, der 2. Präsident der Gesellschaft geworden zu sein. Er hat große Freude an diesem Auftrag gehabt, und er hätte nicht glücklicher über seine Kollegen sein können, durch die er großo Hilfe und Freundlichkeit erfahren hatte. Besonders galt sein Dank Dr. ReNold für dessen wirksame und sichere Hilfe zu jeder Zeit. Die Zahl der Telefongespräche zwischen Genf und Cambridge würden zwar jetzt abnebmen, doch der ,heiße Draht" würde zweifellos jetzt auf die Verbindung Genf-Aarhus verlegt! 
Dr. Younc meinte, daß er ganz besonders froh darüber sei, die Präsidentschaft einem ausgezeichneten Kliniker zu übergeben, und war sicher, daß unter der Führung Dr. LUNDBAEK's die Gesellschaft ihr Wachstum fortsetzen wïrde. Mit großem Beifall beglückwünschte Dr. Young dann Dr. LundBaek zur Präsidentschaft. Der Generalversammlung versicherte Dr. LUNDBAEK mit dem Dank für die jhm übertragene große Ehre, sein Bestes tun zu wollen. Die Gesellschaft war sehr glücklich mit ihrem 2. Präsidenten, der ein gutes Beispiel gegeben habe, und im Namen der Gesellschaft dankte Dr. LuNDBAEK herzlichst Dr. Young für die Jahre des Dienstes und des Vorbildes. Die Ausführungen des Präsidenten wurden mit langem Beifall begrüßt.
Schließlich sagte Dr. LundBaEk, er hätte keine neue Präsidialpolitik anzukündigen. Er gab der Hoffnung Ausdruck, daß die Gesellschaft im selben Geist fortbestehen würde, so überzeugend wie zur Zeit der Gründung, und namentlich wie durch die Veröffentlichung der Diabetologia und die Einrichtung der jährlichen Versammlungen zum Ausdruck gebracht wurde, die immer wieder interessant, sachlich und ohne große Zeremonien verliefen.

In all dieser Hinsicht würde er fortfahren, auf die unbezahlbare Hilfe der Vorstandsmitglieder der Gesellschaft zu baúen, im besonderen auf ihren unermüdlichen Sekretär, Dr. Renowo.

Dr. K. Lundabaek

Präsident

Dr. A.E. RENOLD Sekretär

Responsible for the text: Prof. Dr. K. OBERdisse, II. Med. Klinik u. Poliklinik der Universität, 4000 Düsseldorf, Moorenstr. 5. Responsible for advertisements: Edgar SeIDLen, 1 Berlin 33 (Wilmersdorf), Heidelberger Platz 3. Springer-Verlag, Berlin, Heidelberg, New York.

Printed in Germany by Buchdruckerei Georg Appl, Wemding/Schwaben.

Copyright (C) by Springer-Verlag Berlin - Heidelberg 1969 